\title{
Adverse events related to thoracentesis and chest tube insertion: evaluation of the national collection of subject safety incidents in Japan
}

\author{
Tadashi Kamio $^{1}$ ( $\cdot$ Yusuke lizuka $^{2} \cdot$ Hiroshi Koyama $^{1} \cdot$ Kiyomitsu Fukaguchi $^{1}$
}

Received: 16 October 2020 / Accepted: 7 December 2020 / Published online: 2 January 2021

(c) Springer-Verlag GmbH Germany, part of Springer Nature 2021

\begin{abstract}
Purpose Thoracentesis and chest tube insertion are procedures commonly performed in routine clinical practice and are considered mandatory skills for all physicians. Adverse events secondary to these procedures have been widely reported; however, epidemiology data concerning life-threatening events associated with these procedures are lacking.

Methods We retrospectively analyzed data from the Japan Council for Quality Health Care open database regarding subject safety incidents involving thoracentesis and chest tube insertion. The adverse events extracted from the database included only events associated with thoracentesis and chest tube insertion reported between January 2010 and April 2020.

Results We identified 137 adverse events due to thoracentesis or chest tube insertion. Our analysis also revealed at least 15 fatal adverse events and 17 cases of left/right misalignment. Not only resident doctors but also physicians with 10 years or more of clinical experience had been mentioned in these reports. The most common complications due to adverse events were lung injury (55\%), thoracic vascular injury (21\%), and liver injury (10\%). Surgical treatment was required for $43(31 \%)$ of the 137 cases, and the mortality risk was significantly higher for thoracic vascular injury than for other complications $(p=0.02)$. Conclusion We identified at least 15 fatal adverse events and 17 cases of left/right misalignment over a 10-year period in the Japan Council for Quality Health Care open database. Our findings also suggest that care should be taken to avoid thoracic vascular injury during chest tube insertion and that immediate intervention is required should such an injury occur.
\end{abstract}

Keywords Adverse events · Thoracentesis · Chest tube insertion · Complications

\section{Introduction}

Thoracentesis and chest tube drainage are common surgical procedures performed in routine clinical practice. These procedures help to remove air, blood, purulent material, or other body fluids foreign to this space and to restore the mechanical function of the lung. Therefore, the Advanced Trauma Life Support program teaches the insertion of chest tubes as an essential and a life-saving skill [1]. The chest tube

Tadashi Kamio

tadashi-kamio@mail.goo.ne.jp

1 Division of Critical Care, Shonan Kamakura General Hospital, 1370-1 Okamoto, Kamakura, Kanagawa 247-8533, Japan

2 Department of Anesthesiology and Critical Care Medicine, Jichi Medical University Saitama Medical Center, 1-847 Amanuma-cho, Omiya-ku, Saitama-shi, Saitama 330-8503, Japan should be inserted first to avoid creating an iatrogenic pneumothorax and bleeding; however, adverse events secondary to this procedure have been widely reported [2-7]. The incidence of pneumothorax due to thoracentesis is reportedly about $0.6-4 \%$, and chest tube complications have been reported most frequently in trauma, with an incidence of $2-25 \%$ [8-11]. These adverse events are also associated with significant morbidity and occasional mortality because of cardiac or vascular injury, respiratory distress secondary to pneumothorax, cardiac dysrhythmia, or esophageal injuries. Nevertheless, epidemiology data for life-threatening adverse events associated with thoracentesis and chest tube insertion are still lacking.

In the error reporting systems method, adverse events witnessed or caused by healthcare providers should be reported via structured data collection systems. A clinical incident/accident reporting system is a fundamental tool for improving the safety and quality of care for patients and providing valuable information for medical facilities [12]. 
It is important to share these data so that lessons can be learnt effectively from minor incidents, near-misses, and major accident investigations, which support the work of other organizations to prevent more common and persistent types of patient safety incidents that would rarely be seen in a single institution. In general, life-threatening adverse events associated with thoracentesis and chest tube insertion are rare; therefore, quantitative and qualitative analysis of medical adverse events associated with thoracentesis and chest tube insertion is important. Several studies have used data from incident reporting systems regarding procedures such as insertion of a central vascular catheter, intubation, and anesthesia [13-16], However, to the best of our knowledge, there has been no investigation on thoracentesis and chest tube insertion using incident reporting system data.

According to the World Health Organization Draft Guidelines for Adverse Event Reporting and Learning Systems, the most important goal and measure of success of a reporting system is the use of the results of incident analyses to formulate improvement measures and recommendations for changes in healthcare systems [17]. The Japan Council for Quality Health Care Division of Adverse Event Prevention has been collecting information on medical near-misses and adverse events to prevent adverse events and to promote patient safety since 2004 [18].

Therefore, we analyzed the incident and accident data from a nationwide collection of subject safety incidents involving thoracentesis and chest tube insertion. The primary aim of this study was to compile the epidemiological data on accidents involving thoracentesis and chest tube insertion. A secondary aim was to determine the risk factors for a fatal adverse event.

\section{Methods}

\section{Description of the database}

Since 2004, the Japan Council for Quality Health Care Division of Adverse Event Prevention has been undertaking various activities and projects, including collection of information on medical near-misses and adverse events and evaluation of the quality of medical services provided at hospitals in response to growing concerns about patient safety. As of March 31, 2020, 1531 hospitals and healthcare facilities in Japan (approximately $20 \%$ of the total number of hospitals in the country) were registered with this project. The information gathered concerning medical adverse events is compiled into quarterly and annual reports, wherein the total figures for events and an analysis are also published. These reports are made available to a wide range of individuals and organizations, including medical professionals, members of the public, and government bodies. Since 2010, annual reports have been available to interested parties on the project's Web site (www.med-safe.jp/contents/english/index $. \mathrm{html})$. Regarding medical adverse events, participation in this program is mandatory for tertiary teaching (university, national, and advanced treatment) hospitals; however, other medical institutions have voluntarily joined this project. As of March 31, 2020, 274 hospitals were required by law to report the medical adverse events. There are approximately 13,500 inpatient beds in mandatory reporting medical organizations; therefore, at least 10,000 inpatients per day have been covered for medical adverse event information.

Medical adverse events subject to reporting are as follows: (a) apparent errors in the treatment or management resulting in death, mental or physical disability, need for unexpected treatment, treatment to an unexpected extent, or other medical procedure; (b) unapparent errors in treatment or management resulting in death, mental or physical disability, need for unexpected treatment, treatment to an unexpected extent, or other medical procedures (including events possibly associated with the treatment or management provided; limited to unexpected events); and (c) events other than those described in (a) and (b) with information conducive to the prevention of medical adverse events and their recurrence at medical institutions.

This study was approved by the Institutional Review Board at Shonan Kamakura General Hospital. The hospital review committee waived the need for review in view of the anonymity of the data collected from the database and lack of any link to individual patient information.

\section{Database search methods}

The adverse events extracted from the database included only those associated with thoracentesis and chest tube insertion reported between January 2010 and April 2020. Since this database is only available in Japanese, Japanese words meaning "thoracentesis" and "chest tube insertion" were used for data extraction. The database was searched for all events related to thoracentesis and chest tube insertion, and a free text search was conducted to identify procedurespecific events, such as pneumothorax, bleeding, hemothorax due to perforation of an intercostal artery, and cardiogenic shock in response to compression of a chest tube. In the event of a duplicate report, the data were integrated.

We collected the following data from the database: patient sex and age, location, number of years of clinical experience on the part of the primary operator, complications, additional treatment needed, and the reporter's assessment regarding the possibility of residual disability. Based on the level of harm anticipated by the reporter, the incidents were then classified as follows: (a) low potential for residual disability, (b) high potential for residual 
disability, (c) event resulted in death, and (d) unknown. Two authors (TK and YI) independently reviewed the incident reports to determine their eligibility and classified them by reviewing the free text description submitted at the time of the incident. In view of our focus being on the adverse events associated with thoracentesis and chest tube insertion, we excluded adverse events involving chest surgery and other procedures, such as bronchoscopy, central venous catheterization, lung biopsy, and liver biopsy.

\section{Statistical analyses}

We examined the differences between the two groups ("event not leading to subject's death" and "event resulted in subject's death") for the following variables: operator's clinical experience, adverse events, and incorrect site procedure. Observed and expected numbers of events in the two groups were compared using the Pearson Chi-square or Mann-Whitney $U$ test, as appropriate. All statistical analyses were performed with EZR, which is a graphical user interface for R (The R Foundation for Statistical Computing, Vienna, Austria). More precisely, it is a modified version of $\mathrm{R}$ commander designed to add statistical functions frequently used in biostatistics [19]. All tests were two-sided. Differences with $P$ value $<0.05$ were considered statistically significant.

\section{Results}

A search of incident reports containing the terms "thoracentesis" and "chest tube insertion" identified a total of 687 cases. Reports that were unrelated to the procedures of interest and those that did not meet our inclusion criteria were excluded, leaving 137 cases for inclusion in the analysis (Fig. 1).

Thirty-one of the 137 adverse events involved thoracentesis, and 106 were related to chest tube insertion. An outline of the reports is shown in Table 1. Fifty-nine percent (81/137) procedures were performed in a general ward. The most common complications due to adverse events were lung injury (55\% [75/137]), followed by thoracic vascular injury (21\% [29/137]), liver injury (10\% [14/137]), structural cardiac injury (4\% [5/137]), splenic injury (4\% [5/137]), and misplacement into the abdominal cavity (4\% [6/137]). Indications for chest tube insertion and thoracentesis included pleural effusion (36\% [49/137]), primary pneumothorax (24\% [33/137]), post-surgical care (18\% [25/137]), empyema (14\% [19/137]), traumatic pneumothorax or hemothorax (1\% [2/137]), and others (7\% [9/137]). In our study, the most frequent thoracic vascular injury was in intercostal arteries (66\% [19/29]), followed by chest wall (14\% [4/29]), great vessels $(14 \%$ [4/29]), pulmonary arteries (3\% [1/29]), and unknown (3\% [1/29]). There were 17 cases of left/right misalignment during a procedure; three of these occurred during thoracentesis and 14 during chest tube insertion.
Fig. 1 Flow diagram showing the inclusion and exclusion of incident reports. The flow diagram shows the details for database search and the number of reports screened. Reports that were unrelated to the procedures of interest and those that did not meet our inclusion criteria were excluded
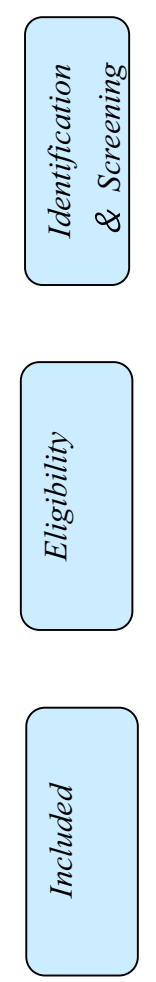

In total, 550 reports were excluded after free text review for the following reasons:

Adverse events associated with other procedures $(n=152)$

Reports of accidental or self-removal of drain/tube $(n=67)$

Adverse events during an operation $(\mathrm{n}=37)$

Reports not associated with the procedures $(n=294)$ 
Table 1 Characteristics of the adverse events

\begin{tabular}{|c|c|c|c|}
\hline & Total & Thoracentesis & Chest tube insertion \\
\hline Reports, $n$ & 137 & 31 & 106 \\
\hline \multicolumn{4}{|l|}{ Patient age, years, $n(\%)$} \\
\hline $0-9$ & $2(2 \%)$ & 0 & $2(2 \%)$ \\
\hline $10-19$ & $2(2 \%)$ & 0 & $2(2 \%)$ \\
\hline $20-69$ & $68(50 \%)$ & $17(55 \%)$ & $51(48 \%)$ \\
\hline $70-79$ & $36(26 \%)$ & $7(23 \%)$ & $29(27 \%)$ \\
\hline$\geq 80$ & $29(21 \%)$ & $7(23 \%)$ & $22(20 \%)$ \\
\hline \multicolumn{4}{|l|}{ Setting of care, $n(\%)$} \\
\hline General ward & $81(59 \%)$ & $21(68 \%)$ & $60(57 \%)$ \\
\hline ICU/NICU & $23(17 \%)$ & $4(13 \%)$ & $19(18 \%)$ \\
\hline Emergency department & $9(7 \%)$ & $1(3 \%)$ & $8(8 \%)$ \\
\hline Outpatient clinic & $7(5 \%)$ & $4(13 \%)$ & $3(3 \%)$ \\
\hline Other & $17(12 \%)$ & $1(3 \%)$ & $16(15 \%)$ \\
\hline \multicolumn{4}{|l|}{ Indications, $n(\%)$} \\
\hline Pleural effusion & $49(36 \%)$ & $22(71 \%)$ & $27(25 \%)$ \\
\hline Primary pneumothorax & $33(24 \%)$ & 0 & $33(31 \%)$ \\
\hline Post-surgical care & $25(18 \%)$ & $4(13 \%)$ & $21(20 \%)$ \\
\hline Empyema & $19(14 \%)$ & $1(3 \%)$ & $18(17 \%)$ \\
\hline Traumatic pneumothorax or hemothorax & $2(1 \%)$ & $1(3 \%)$ & $1(1 \%)$ \\
\hline Others & $9(7 \%)$ & $3(10 \%)$ & $6(6 \%)$ \\
\hline \multicolumn{4}{|l|}{ Adverse events, $n(\%)$} \\
\hline Lung injury & $75(55 \%)$ & $20(65 \%)$ & $55(52 \%)$ \\
\hline Thoracic vascular injury & $29(21 \%)$ & $10(32 \%)$ & $19(18 \%)$ \\
\hline Structural cardiac injury & $5(4 \%)$ & $1(3 \%)$ & $4(4 \%)$ \\
\hline Liver injury & $14(10 \%)$ & 0 & $14(13 \%)$ \\
\hline Spleen injury & $5(4 \%)$ & 0 & $5(5 \%)$ \\
\hline Misplacement into the abdominal cavity & $6(4 \%)$ & 0 & $6(6 \%)$ \\
\hline Others & $3(2 \%)$ & 0 & $3(3 \%)$ \\
\hline Wrong site procedure, $\mathrm{n}(\%)$ & $17(12 \%)$ & $3(10 \%)$ & $14(13 \%)$ \\
\hline
\end{tabular}

$I C U$ intensive care unit; NICU neonatal intensive care unit
Figure 2 shows a summary of the incident reports and the years of clinical experience of the operators. Although operators with 3-10-years' experience after graduation tended to report more incidents, many were also reported by physicians with at least 10 years of clinical experience.

Table 2 summarizes the adverse events according to the procedure performed and additional treatments required for associated complications. Thirty-one percent (23/75) of the 75 cases of lung injury required surgery. Ventilator management was required for complications involving lung injury in $32 \%(24 / 75)$ patients. Surgical treatment was required for all five cases of structural cardiac injury. Thirty-six percent $(5 / 14)$ of the patients with liver injury received endovascular treatment, and 29\% (4/14) required surgical hemostasis.

Figure 3 shows the level of harm in the patients who experienced adverse events. There was no residual impairment in 79\% (109/137) patients and some residual impairment in $8 \%$ (11/137) patients. Eleven percent (15/137) of the patients died. Table 3 compares the median number of years of the operator's clinical experience, number of adverse events, and number of wrong site procedures according to whether or not the adverse event proved fatal. The death rate was significantly higher for thoracic vascular injury $(p=0.02)$. No significant differences were observed in other parameters.

\section{Discussion}

Chest tube insertion is a procedure commonly performed by the residents and fellows and is classified as a mandatory skill for all physicians. In this study, we identified 137 adverse events due to thoracentesis or chest tube insertion between 2010 and 2020 in the Japan Council for Quality Health Care open database. Our analysis also revealed that at least 15 fatal adverse events and 17 cases of left/right misalignment occurred in Japan during the study period. Not only resident doctors but also physicians with at least 10 years of clinical experience were the operators in these 
Fig. 2 Summary of number of incident reports and years of clinical experience of the operators

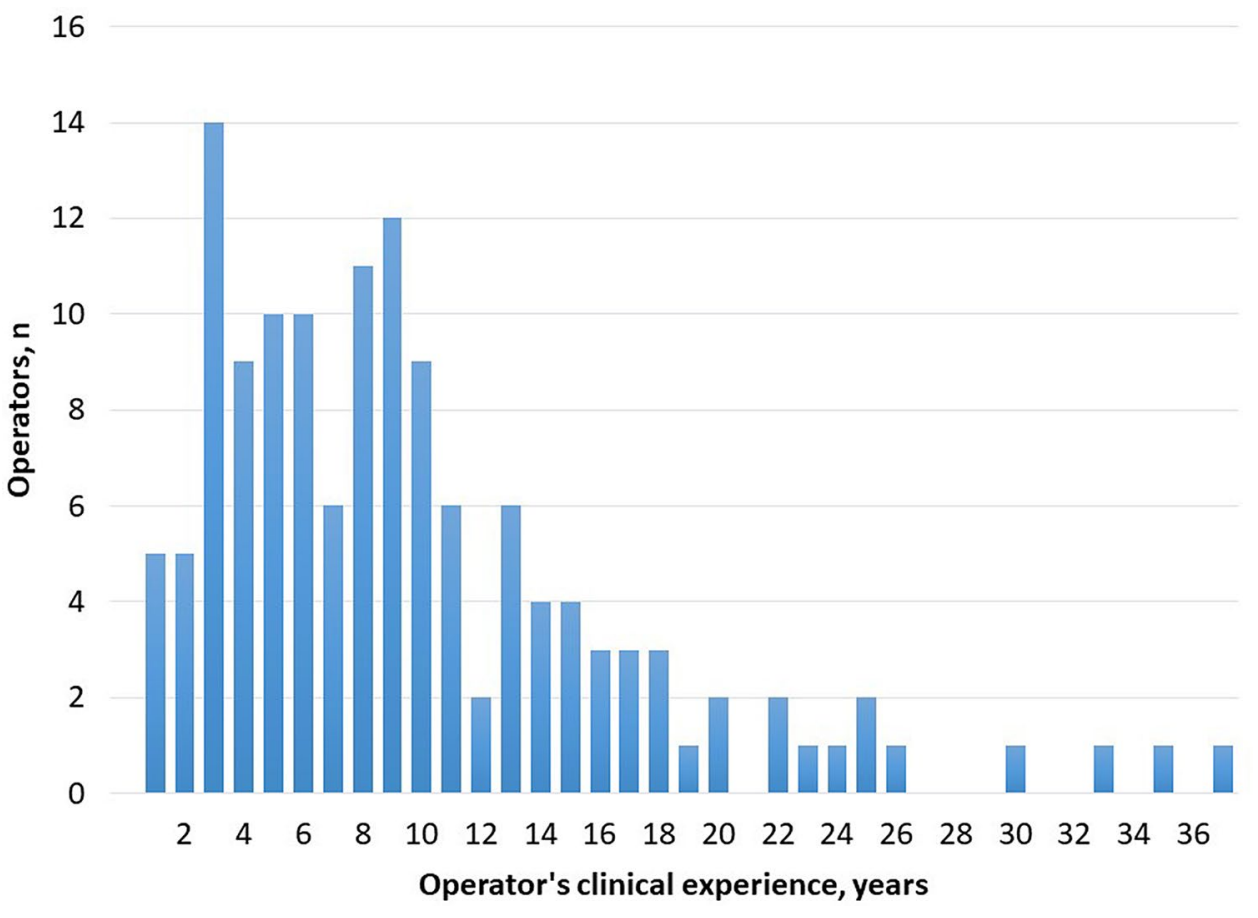

Table 2 Summary of adverse events due to procedures and additional treatments

\begin{tabular}{|c|c|c|c|c|c|}
\hline & \multirow{2}{*}{$\begin{array}{l}\text { Total number of } \\
\text { adverse events }\end{array}$} & \multicolumn{3}{|c|}{ Additional treatment, $n(\%)$} & \multirow{2}{*}{$\begin{array}{l}\text { Mechanical } \\
\text { ventilation }\end{array}$} \\
\hline & & $\begin{array}{l}\text { Trans-arterial } \\
\text { embolization }\end{array}$ & Surgical repair & Transfusion & \\
\hline Lung injury & 75 & 0 & $23(31 \%)$ & $10(13 \%)$ & $24(32 \%)$ \\
\hline Thoracic vascular injury & 29 & $9(31 \%)$ & $8(28 \%)$ & $25(86 \%)$ & $16(55 \%)$ \\
\hline Structural cardiac injury & 5 & 0 & $5(100 \%)$ & $4(80 \%)$ & $5(100 \%)$ \\
\hline Liver injury & 14 & $5(36 \%)$ & $4(29 \%)$ & $5(36 \%)$ & $5(36 \%)$ \\
\hline Splenic injury & 5 & 0 & $2(40 \%)$ & $1(20 \%)$ & $3(60 \%)$ \\
\hline Misplacement into abdominal cavity & 6 & $1(17 \%)$ & 0 & 0 & 0 \\
\hline
\end{tabular}

reports. The most common complications due to adverse events were lung injury (55\%), followed by thoracic vascular injury $(21 \%)$ and liver injury (10\%). Surgical treatment was required for 43 (31\%) of the 137 cases, and the risk of death was significantly higher for thoracic vascular injury than for other complications. Our results suggest that extreme care should be taken to avoid thoracic vascular injury when inserting a chest tube and immediate intervention is required if such an injury occurs.

Thoracentesis and chest tube insertion can be associated with the life-threatening complications, including pneumothorax, hemorrhage, and organ injury; however, there is a paucity of high-quality recent literature on these procedurerelated complications [20]. To the best of our knowledge, this is the first study to analyze data on the adverse events associated with thoracentesis and chest tube insertion using a nationwide collection of subject safety incidents. Harris et al. used a questionnaire survey to evaluate current practice and any adverse incidents related to chest drains among 198 chest physicians in the UK from 2003 to 2008 and found 31 cases of misplacement of an intercostal chest drain that was fatal in seven cases [21]. We similarly identified at least 15 fatal adverse events over a period of 10 years in Japan. Moreover, we found that thoracic vascular injury secondary to thoracentesis or chest tube insertion had the highest mortality risk. Thoracic vascular injury involves damage to an intercostal artery, chest wall, or pulmonary artery. Chemelli et al. demonstrated a 30-day mortality rate of $30.8 \%$ in patients who had sustained an iatrogenic intercostal artery injury [22]. Critically ill patients who need chest tube insertion often experience more problems in coping with arterial injury and clotting disorders, which are often induced 


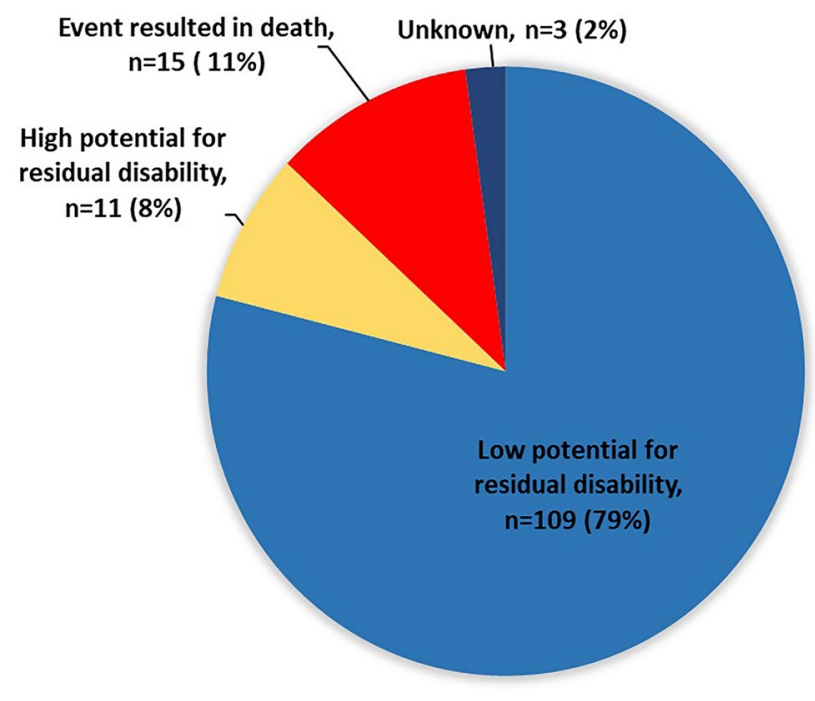

Fig. 3 The details of level of harm in patients who experienced adverse events

by massive hemorrhage, and may be life-threatening. Our results suggest that the patients with thoracic vascular injury secondary to thoracentesis or chest tube insertion should be considered for early transfusion and emergent transcatheter arterial embolization or surgical repair [22-24]. Transcatheter arterial embolization in patients with intercostal arteries' injury is effective; however, this technique may be accompanied by a complete and permanent spinal cord injury [25]. The procedure is approached with caution when embolization involves branches arising from the 10th to 12 th thoracic segments.
A previous study [21] reported that the misplaced drains were most often inserted into the liver, followed by the peritoneal space, heart, spleen, subclavian vessels, colon, and inferior vena cava. This finding indicates a need for close attention to liver injury associated with thoracentesis and chest tube insertion, despite a comprehensive review describing liver injury associated with placement of a thoracostomy tube as uncommon [9]. Hepatic injury secondary to chest tube insertion may result in life-threatening complications requiring emergency surgery; therefore, it is crucial to identify a potentially high-risk patient or situation. Further research is needed to identify gaps in knowledge.

Our findings and those of the previous surveys $[21,26]$ also indicated that the adverse incidents related to chest drains are not only caused by residents and fellows but also by senior doctors. In general, resident doctors have less experience in chest tube insertion techniques than their senior counterparts. Insertion of a central venous catheter by a physician with extensive experience is half as likely to result in a mechanical complication as it would be when inserted by a less skilled doctor $[27,28]$. On the other hand, overconfidence that comes with years of clinical experience may lead to accidents. The Quality in Australian Health Care Study identified 2351 adverse events related to hospitalization, of which $10.9 \%$ were categorized as "a lack of care and attention or failure to attend the patient" [29]. Given that many doctors tend to overestimate their skills and abilities, new policies, protocols, and technologies are needed to aid the cognitive activities of the clinicians [30]. Unfortunately, we could not obtain information concerning the use of echography; however, echography should be used to mark the entry point for all chest tubes so as to prevent incorrect
Table 3 Comparison of parameters between fatal and non-fatal adverse events

\begin{tabular}{llll}
\hline & \multicolumn{2}{l}{ Level of harm } & $P$ value \\
\cline { 2 - 3 } & Non-fatal event & Fatal event & \\
\hline Total number & 122 & 15 & \\
$\begin{array}{l}\text { Operator's clinical experience, years, median } \\
\text { (IQR) }\end{array}$ & $8(5-13)$ & $8(3.5-12.5)$ & $0.56^{*}$ \\
Adverse event, $n(\%)$ & & & \\
Lung injury & $70(57 \%)$ & $5(33 \%)$ & $0.10^{\dagger}$ \\
Thoracic vascular injury & $22(18 \%)$ & $7(47 \%)$ & $0.02^{\dagger}$ \\
Structural cardiac injury & $4(3 \%)$ & $1(7 \%)$ & $0.45^{\dagger}$ \\
Liver injury & $12(10 \%)$ & $2(13 \%)$ & $0.65^{\dagger}$ \\
Splenic injury & $5(4 \%)$ & 0 & \\
Misplacement into abdominal cavity & $6(5 \%)$ & 0 & \\
Other & $3(2 \%)$ & 0 & $0.69^{\dagger}$ \\
Wrong site procedure, $n(\%)$ & $16(13 \%)$ & $1(7 \%)$ & \\
\hline
\end{tabular}

$I Q R$ interquartile range

*Mann-Whitney $U$ test

${ }^{\dagger}$ Chi-square test 
placement and reduce the risk of accidental organ injury associated with the procedure [31].

In 2001, Ken Kizer of the National Quality Forum was credited with coining the term "never event" in reference to medical errors that should never occur in a patient. Wrong site surgery or other invasive procedure has consistently been the commonest "never events." It is estimated that the rate of wrong site surgery varies widely, reportedly ranging from 0.09 to 4.5 per 10,000 surgeries performed [32]. We identified 17 wrong site thoracentesis and chest tube insertion errors over a 10-year period in Japan. Miller et al. examined a root cause analysis database for reported wrong-side thoracentesis from the National Center for Patient Safety database in ambulatory clinics and hospital units reported from 2004 to 2011 [33]. They identified 14 cases of wrongside thoracentesis and found that communication failure, unawareness of existing policy, and a training deficit were the most common root causes of wrong-side thoracentesis. Moreover, it is difficult to diagnose pneumothorax and pleural effusion on a physical examination. The natural symmetry of the body may contribute to confusion regarding procedural laterality and patient positioning [34]. Although we could not estimate the incidence (proportion or rate), it is important to demonstrate these data to draw attention to wrong site procedures.

Similar to all retrospective analyses, our study has several limitations. First, despite using a uniform method of data collection, the quality of the data in this database was variable as a result of variations in the data submitted by the staff of individual facilities. Although this database has not been validated, an English-language version of the annual report is created each year since 2005 , and the quarterly and annual reports contain large volumes of information. Second, more than 1000 hospitals and healthcare facilities in Japan have joined this nationwide healthcare database; however, the reporting of such adverse events is dependent on each healthcare professional, and our results may not be fully representative of the true situation. The types of errors that are reported will differ not just on the actual number/type of true errors occurring, but also on the ability to recognize and report them in the first place. Therefore, our results may probably underestimate the actual occurrences of subject safety incidents. However, we extracted all data from the database and conducted a free text search to obtain precise information. Third, the incidence rate is defined as the frequency of new occurrences of medical disorders in the specific population at risk of the medical disorders arising in a given period of time; however, similar to the past reports, it is impossible to calculate the incidence in this study because the actual denominator is unknown. However, some of these complications can be "never event"; thus, it is important to show the epidemiology data for life-threatening adverse events associated with thoracentesis and chest tube insertion. Because of the limitations of simple clinical case reports when extrapolating to the entire population, epidemiological data remain an important evidence for a risk assessment.

\section{Conclusions}

Thoracentesis and chest tube insertion are not without risk, and a multitude of complications is associated with these procedures, some of which are potentially fatal. We identified that at least 15 fatal adverse events and 17 cases of left/right misalignment had occurred over 10 years in the Japan Council for Quality Health Care open database. Our findings highlight the need for extreme care when inserting a chest tube to avoid thoracic vascular injury as well as the need for urgent intervention if such an injury occurs.

Acknowledgements Dr. Tadashi Kamio takes responsibility for the content of this manuscript, including the data, analysis, draft, and final version.

Author contributions TK is responsible for the content of the manuscript, including figures and tables. TK and YI independently reviewed the incident reports to determine eligibility and classified them by reviewing the free text description submitted at the time of the incident. HK and KF contributed to the preparation and revision of the manuscript. All authors read and approved the final manuscript.

Funding The author(s) received no financial support for the research, authorship, and/or publication of this article.

Availability of data and material Medical event information and medical near-miss event information from 2010 are available via the project's Web site (www.med-safe.jp/contents/english/index.html). The open database contains annual reports and materials (in Japanese).

Code availability Not applicable.

\section{Compliance with ethical standards}

Conflicts of interest The authors declare that they have no competing interests.

Ethical approval This study was approved by the Institutional Review Board at Shonan Kamakura General Hospital. The hospital review committee waived the need for review in view of the anonymity of the data collected from the database and lack of any link to individual patient information.

Consent to participate Not applicable.

Consent for publication Not applicable.

\section{References}

1. Bowness JS, Nicholls K, Kilgour PM, Ferris J, Whiten S, Parkin I, et al. Finding the fifth intercostal space for chest drain insertion: guidelines and ultrasound. Emerg Med J. 2015;32(12):951-4.

2. Mehrotra AK, Feroz A, Dawar S, Kumar P, Singh A, Khublani TK. Diaphragmatic rupture precipitated by intercostal chest tube 
drainage in a patient of blunt thoraco-abdominal trauma. Lung India. 2016;33(1):85-7.

3. Shechtman L, Shrem M, Kleinbaum Y, Bornstein G, Gilad L, Grossman C. Incidence and risk factors of pneumothorax following pre-procedural ultrasound-guided thoracentesis. J Thorac Dis. 2020;12(3):942-8.

4. Kong VY, Oosthuizen GV, Sartorius B, Keene C, Clarke DL. An audit of the complications of intercostal chest drain insertion in a high volume trauma service in South Africa. Ann R Coll Surg Engl. 2014;96(8):609-13.

5. Kong VY, Clarke DL. The spectrum of visceral injuries secondary to misplaced intercostal chest drains: experience from a high volume trauma service in South Africa. Injury. 2014;45(9):1435-9.

6. Gordon CE, Feller-Kopman D, Balk EM, Smetana GW. Pneumothorax following thoracentesis: a systematic review and metaanalysis. Arch Intern Med. 2010;170(4):332-9.

7. Varghese S, Slottosch I, Saha S, Wacker M, Awad G, Wippermann J, et al. Surgical management of iatrogenic left ventricle perforation by chest tube insertion. Ann Thorac Surg. 2019;108(6):e405-7.

8. Menger R, Telford G, Kim P, Bergey MR, Foreman J, Sarani B, et al. Complications following thoracic trauma managed with tube thoracostomy. Injury. 2012;43(1):46-50.

9. Kwiatt M, Tarbox A, Seamon MJ, Swaroop M, Cipolla J, Allen C, et al. Thoracostomy tubes: a comprehensive review of complications and related topics. Int J Crit Illn Inj Sci. 2014;4(2):143-55.

10. Sagar AES, Landaeta MF, Adrianza AM, Aldana GL, Pozo L, Armas-Villalba A, et al. Complications following symptom limited thoracentesis using suction. Eur Respir J. 2020;56(5):1902356.

11. Ault MJ, Rosen BT, Scher J, Feinglass J, Barsuk JH. Thoracentesis outcomes: a 12-year experience. Thorax. 2015;70(2):127-32.

12. Hutchinson A, Young TA, Cooper KL, McIntosh A, Karnon JD, Scobie S, et al. Trends in healthcare incident reporting and relationship to safety and quality data in acute hospitals: results from the national reporting and learning system. Qual Saf Health Care. 2009;18(1):5-10.

13. Szekely SM, Webb RK, Williamson JA, Russell WJ. The Australian Incident Monitoring Study. Problems related to the endotracheal tube: an analysis of 2000 incident reports. Anaesth Intens Care. 1993;21(5):611-6.

14. Domino KB, Bowdle TA, Posner KL, Spitellie PH, Lee LA, Cheney FW. Injuries and liability related to central vascular catheters: a closed claims analysis. Anesthesiology. 2004;100(6):1411-8.

15. Hohenstein C, Schultheis K, Winning J, Rupp P, Fleischmann T. Critical incidents in preclinical emergency airway management: evaluation of the CIRS emergency medicine databank. Anaesthesist. 2013;62(9):720-4.

16. Cheney FW, Domino KB, Caplan RA, Posner KL. Nerve injury associated with anesthesia: a closed claims analysis. Anesthesiology. 1999;90(4):1062-9.

17. Mahajan RP. Critical incident reporting and learning. Br J Anaesth. 2010;105(1):69-75.

18. The Japan Council for Quality Health Care Division of Adverse Event Prevention. http://www.med-safe.jp/contents/english/index .html. (Accessed 01 July 2020)
19. Kanda Y. Investigation of the freely available easy-to-use software 'EZR' for medical statistics. Bone Marrow Transplant. 2013;48(3):452-8.

20. Mao M, Hughes R, Papadimos TJ, Stawicki SP. Complications of chest tubes: a focused clinical synopsis. Curr Opin Pulm Med. 2015;21(4):376-86.

21. Harris A, O'Driscoll BR, Turkington PM. Survey of major complications of intercostal chest drain insertion in the UK. Postgrad Med J. 2010;86(1012):68-72.

22. Chemelli AP, Thauerer M, Wiedermann F, Strasak A, Klocker J, Chemelli-Steingruber IE. Transcatheter arterial embolization for the management of iatrogenic and blunt traumatic intercostal artery injuries. J Vasc Surg. 2009;49(6):1505-13.

23. Holcomb JB, Jenkins D, Rhee P, Johannigman J, Mahoney P, Mehta S, et al. Damage control resuscitation: directly addressing the early coagulopathy of trauma. J Trauma. 2007;62(2):307-10.

24. Holcomb JB, Tilley BC, Baraniuk S, Fox EE, Wade CE, Podbielski JM, et al. Transfusion of plasma, platelets, and red blood cells in a 1:1:1 vs. a 1:1:2 ratio and mortality in patients with severe trauma: the PROPPR randomized clinical trial. JAMA. 2015;313(5):471-82.

25. Ozoilo K, Stein M. Paraplegia complicating embolization for bleeding intercostal artery in penetrating trauma. Injury Extra. 2013;44(8):70-3.

26. Ball CG, Lord J, Laupland KB, Gmora S, Mulloy RH, Ng AK, et al. Chest tube complications: how well are we training our residents? Can J Surg. 2007;50(6):450-8.

27. Sznajder JI, Zveibil FR, Bitterman H, Weiner P, Bursztein S. Central vein catheterization. Failure and complication rates by three percutaneous approaches. Arch Intern Med. 1986;146(2):259-61.

28. Bo-Linn GW, Anderson DJ, Anderson KC, McGoon MD. Percutaneous central venous catheterization performed by medical house officers: a prospective study. Cathet Cardiovasc Diagn. 1982;8(1):23-9.

29. Wilson RM, Harrison BT, Gibberd RW, Hamilton JD. An analysis of the causes of adverse events from the quality in Australian health care study. Med J Aust. 1999;170(9):411-5.

30. Berner ES, Graber ML. Overconfidence as a cause of diagnostic error in medicine. Am J Med. 2008;121(5 Suppl):S2-23.

31. Porcel JM. Pleural ultrasound for clinicians. Rev Clin Esp. 2016;216(8):427-35 (Article in Spanish).

32. Devine J, Chutkan N, Norvell DC, Dettori JR. Avoiding wrong site surgery: a systematic review. Spine (Phila Pa 1976). 2010;35(9 Suppl):S28-36.

33. Miller KE, Mims M, Paull DE, Williams L, Neily J, Mills PD, et al. Wrong-side thoracentesis: lessons learned from root cause analysis. JAMA Surg. 2014;149(8):774-9.

34. Seiden SC, Barach P. Wrong-side/wrong-site, wrong-procedure, and wrong-patient adverse events: are they preventable? Arch Surg. 2006;141(9):931-9. 